\title{
Concordância e correlação entre a perimetria de freqüência dupla e a perimetria azul-amarelo no glaucoma: sem suporte para seletividade
}

\author{
Agreement and correlation bet ween the frequency doubling perimetry and the blue- \\ yellow perimetry in glaucoma:no support for selectivedamage
}

\author{
Leopoldo Magacho' \\ Ricardo Reis ${ }^{2}$ \\ Bernardo Magacho ${ }^{3}$ \\ Marcos Pereira Ávila ${ }^{4}$
}

\footnotetext{
Trabalho realizado no Setor de Glaucoma do Centro de Referência em Oftalmologia da Universidade Federal de Goiás (CEROF/UFG) - Goiânia (GO) - Brasil.

${ }^{1}$ Professor da Pós-graduação da Universidade Federal de Goiás - UFG - Goiânia (GO) - Brasil. Coordenador, Doutor do Setor de Glaucoma do Centro de Referência em Oftalmologia - CEROF da UFG - Goiânia (GO) Brasil.

2 Responsável pelo Setor de Glaucoma da Santa Casa de Misericórdia de São José do Rio Preto - São José do Rio Preto (SP) - Brasil.

${ }^{3}$ Oftalmologista da Pronto Clínica de Olhos de Goiânia - Goiânia (GO) - Brasil.

${ }^{4}$ Professor, Doutor e chefe do CEROF da UFG - Goiânia (GO) - Brasil.

Endereço para correspondência: Leopoldo Magacho. Rua 131, nº 313 - Setor Sul - Goiânia (GO)

CEP 74093-200

E-mail: lmagacho@brturbo.com.br

Recebido para publicação em 07.12.2004

Última versão recebida em 08.11 .2006

Aprovação em 11.12.2006

Os autores não possuem interesse comercial em qualquer um dos produtos citados no texto.
}

\section{RESUMO}

Objetivo: Avaliar a concordância e correlação entre a perimetria de freqüência dupla e a perimetria azul-amarelo no glaucoma. Métodos: Pacientes com glaucoma foram selecionados do Setor de Glaucoma do Centro de Referência em Oftalmologia (CEROF) da Universidade Federal de Goiás, e submetidos aos exames de perimetria de freqüência dupla e perimetria azul-amarelo em ambos os olhos (quando elegíveis) no mesmo dia de maneira aleatória. Foi obtido a correlação (Pearson) entre os índices globais (mean deviation - MD e pattern standard deviation - PSD) e a concordância entre os exames (índice Kappa) para a gravidade do dano glaucomatoso. Resultados: Quarenta e três olhos de 26 pacientes foram incluídos no estudo. Comparando-se o MD $(-4,5 \pm 4,5 \mathrm{~dB}$ para o perimetria de freqüência dupla e $-8,0 \pm 6,8 \mathrm{~dB}$ para o perimetria azulamarelo, $\mathrm{p}<0,001)$ e o PSD $(6,4 \pm 2,8 \mathrm{~dB}$ para o perimetria de freqüência dupla e $5,8 \pm 2,4 \mathrm{~dB}$ para o perimetria azul-amarelo, $\mathrm{p}=0,1)$, verificamos que apenas o MD mostrou diferença estatisticamente significativa entre os grupos. Os índices globais MD e PSD foram altamente correlacionados comparando-se o perimetria de freqüência dupla e o perimetria azulamarelo $(r=0,644, p<0,001$ e $r=0,586, p<0,001$, respectivamente). A concordância entre as classificações da gravidade da perda glaucomatosa mostrou-se significativa (Kappa=0,319, p<0,001). Conclusão: Existe alta correlação entre os índices globais e alta concordância em termos de classificação da gravidade do dano glaucomatoso entre a perimetria azulamarelo e a perimetria de freqüência dupla, refletindo ausência de seletividade no dano glaucomatoso, ou que os instrumentos disponíveis não sejam capazes de isolar as células ganglionares de forma completa.

Descritores: Glaucoma/diagnóstico; Perimetria/métodos; Pressão intra-ocular; Estudo comparativo

\section{INTRODUCÃO}

O glaucoma pode ser caracterizado como uma doença progressiva e irreversível aos tecidos oculares. O exame considerado como "gold standard" ainda é a perimetria acromática. Entretanto, é necessário a perda de cerca de $30 \%$ das células ganglionares retinianas para um defeito detectável de campo visual ${ }^{(1-2)}$.

Nos últimos tempos, novas estratégias têm surgido com a finalidade de se detectar o glaucoma ainda nas fases iniciais. A perimetria azul-amarelo (SWAP - Short Wavelenght Automated Perimetry) testa as células da 
camada parvocelular do corpo geniculado lateral (CGL), sendo postulado a detecção do defeito perimétrico até 5 anos antes da perimetria convencional ${ }^{(3-4)}$. Mais recentemente, foi introduzido no nosso arsenal propedêutico a perimetria de frequiência dupla (FDT), que visa as células da camada magnocelular do CGL, mais sensíveis aos movimentos ${ }^{(5)}$. Estudos também têm mostrado a capacidade do FDT de detectar a lesão perimétrica antes do campo visual branco-branco ${ }^{(6)}$, e alta sensibilidade e especificidade na detecção do dano perimétrico glaucomatoso ${ }^{(7-10)}$.

Em recente estudo, alguns autores ${ }^{(11)}$ mostraram que as vias magnocelular (testada pelo FDT) e parvocelular (perimetria de alta resolução) apresentavam concordância elevada em pacientes com glaucoma, sugerindo que elas podem ser igualmente lesadas, ou que não existe seletividade nos aparelhos testados. O presente estudo tem a finalidade de verificar se o FDT e a SWAP apresentam diferenças detectáveis no exame do paciente glaucomatoso.

\section{MÉTODOS}

Pacientes com glaucoma foram recrutados do Setor de Glaucoma do Centro de Referência em Oftalmologia (CEROF) da Universidade Federal de Goiás após consentimento livre, informado, assinado e aprovação do Comitê de Ética da respectiva instituição.

Todos os pacientes foram submetidos a exame oftalmológico completo, incluindo exame da acuidade visual melhor corrigida (tabela de Snellen ou projetor ajustado para 6 metros), biomicroscopia, tonometria com tonômetro de Goldmann (como parte da consulta para o glaucoma), fundoscopia sob midríase e perimetria computadorizada bilateral (quando possível) caso o exame tivesse sido realizado há mais de seis meses, ou se o paciente sofreu alguma intervenção cirúrgica no período entre o último teste e a aplicação do questionário. Além disso, foi solicitado o SWAP (Humphrey Systems, Dublin, CA, EUA) e o exame de FDT, estratégia C-20, limiar completo (Welch Allyn Skaneateles, New York, EUA e Zeiss Humphrey Systems, Dublin, CA, EUA), realizados no mesmo dia em ambos os olhos (se elegíveis), e de maneira aleatória para diminuir o efeito fadiga dos exames ${ }^{(12)}$, sempre o olho direito seguido do olho esquerdo. Para o exame de FDT, foi utilizado a estratégia de "screening" em um dos olhos de forma randômica como demonstrativo para diminuir o efeito aprendizado da máquina ${ }^{(13)}$.

Os critérios de inclusão foram: diagnóstico de glaucoma há pelo menos seis meses (presença de pelo menos dois exames de campo visual SITA Standard 24-2 (Humphrey Systems, Dublin, CA, EUA) confiáveis ${ }^{(14)}$ e com alterações características de glaucoma, segundo os critérios propostos por HodappParrish-Anderson (HPA) ${ }^{(15)}$, exame do disco óptico demonstrando lesão típica da doença, ou seja, afinamento localizado ou perda global da rima nervosa, alterações vasculares ${ }^{(16)}$, hemorragia de disco óptico), capacidade de realizar campo visual em pelo menos um dos olhos, acuidade visual melhor corrigida maior ou igual a 20/100 e consentimento livre, informado e assinado em participar do estudo.

Os critérios de exclusão foram: perda ou déficit de visão em qualquer olho de causa não conhecida ou outra além do glaucoma, cirurgia intra-ocular recente ( 3 meses), campo visual SWAP (mesmo para o campo SITA) e/ou FDT (segundo determinação da própria máquina) não confiáveis, pacientes com incapacidade de realizar o campo visual em ambos os olhos, afacia ou qualquer outra alteração à biomicroscopia ou fundoscopia que pudesse interferir no exame de perimetria computadorizada, como degeneração macular relacionada à idade, catarata maior que leve ou descolamento de retina e recusa do paciente em participar do estudo.

No presente estudo, o campo SWAP foi considerado como anormal seguindo os mesmos critérios do campo SITA, enquanto que para o campo FDT, a presença de uma área afetada, independentemente da localização ou profundidade foi definido como alteração. Além disso, os campos SAP foram classificados como iniciais, moderados ou graves por um examinador experiente e mascarado (LM).

Os cálculos estatísticos foram realizados pelo software SPSS (SPSS Inc, Chicago, IL, EUA), versão 11.5. Estabelecendo-se o erro alfa em 0,05 , e o poder do teste em $80 \%$, o número estimado de pacientes necessários para se detectar correlações altamente significativas $(r>0,5)$ foi de 30 . A normalidade das amostras foi obtida com o teste de Kolmogorov-Smirnov. A correlação entre os índices globais (mean deviation - MD e pattern standard deviation - PSD) foi obtida com a correlação de Pearson. A concordância no diagnóstico do glaucoma, e para a gravidade do mesmo foi obtida com o índice Kappa, sendo sua significância testada com o teste de Qui-quadrado. O teste T de Student pareado foi utilizado para verificar se houve diferença entre os índices globais do FDT e SWAP nos olhos estudados. Valores de p menores que 0,05 foram considerados como significativos.

\section{RESULTADOS}

Quarenta e três olhos de 26 pacientes foram incluídos no estudo, sendo 35 mulheres e 8 homens, com idade média de $49,9 \pm 14,2$ anos.

Baseando-se na perimetria acromática, o MD médio foi de $-7,0 \pm 4,8 \mathrm{~dB}$, enquanto o PSD médio foi de $6,6 \pm 4,1 \mathrm{~dB}$. Utilizando-se a classificação de HPA ${ }^{(15)}$ para o mesmo campo, obtivemos 19 campos com dano inicial, 19 com dano moderado e apenas 5 campos foram considerados como graves.

Comparando-se o MD $(-4,5 \pm 4,5 \mathrm{~dB}$ para o FDT e $-8,0 \pm 6,8 \mathrm{~dB}$ para o SWAP, $\mathrm{p}<0,001)$ e o PSD $(6,4 \pm 2,8 \mathrm{~dB}$ para o FDT e $5,8 \pm$ $2,4 \mathrm{~dB}$ para o SWAP, $\mathrm{p}=0,1)$ verificamos que apenas o MD obteve diferença estatisticamente significativa entre os grupos. Os índices globais MD e PSD foram altamente correlacionados comparando-se o FDT e o SWAP ( $r=0,644$, $p<0,001$ e r=0,586, 
$\mathrm{p}<0,001$, respectivamente). A concordância entre as classificações da gravidade da perda glaucomatosa mostrou-se significativa (Kappa $=0,319, \mathrm{p}<0,001$ ), mais evidenciado na tabela.

\section{DISCUSSÃO}

Porque o dano clinicamente detectável na perimetria acromática apenas é percebido após a perda de cerca de $30 \%$ das células ganglionares retinianas ${ }^{(1)}$, novas estratégias para detecção da lesão precoce do glaucoma foram oferecidas como parte do arsenal terapêutico para a doença.

A perimetria de cores, ou perimetria azul-amarelo, visa um subgrupo de células ganglionares da retina que seriam precocemente danificadas no glaucoma. Estudos evidenciaram lesões reprodutíveis até 5 anos antes do aparecimento do dano à perimetria branco-branco ${ }^{(4)}$. Entretanto, é possível que a detecção precoce proposta na perimetria de cores possa ter outras explicações, inclusive pela diminuição do contraste gerado pelos estímulos e fundo da cúpula de cores diferentes do preto e branco. Além disso, por ser uma estratégia mais longa, pode provocar uma maior fadiga nos pacientes, o que também pode influenciar nos resultados. Com o advento da estratégia SITA, esse inconveniente, pelo menos em parte, deverá ser melhorado.

A perimetria de freqüência dupla, testa um outro subtipo de células, mais sensíveis aos movimentos, as magnocelulares, também supostamente lesadas mais precocementes na patogênese do glaucoma ${ }^{(6-7)}$. Estas células, maiores, seriam inicialmente lesadas por serem relativamente mais numerosas nas regiões do disco óptico inicialmente atingidas no glaucoma. Seriam ainda mais sensíveis aos aumentos da pressão intra-ocular.

Como o FDT e o SWAP são estratégias distintas, é esperado que não ocorra concordância entre elas no diagnóstico do glaucoma, especialmente em pacientes com dano glaucomatoso inicial. A amostra do presente estudo reflete uma população glaucomatosa com o dano perimétrico, no geral, de inicial para moderado (MD médio de $-7,0 \pm 4,8 \mathrm{~dB}$ ). Apesar das estratégias terem demonstrado diferença estatisticamente significativas em relação ao $\mathrm{MD}$ ( $\mathrm{p}<0,001$ ), foi evidenciado uma associação linear importante entre as duas, tanto para o MD, quanto para o $\operatorname{PSD}(r=0,644, p<0,001$ e $r=0,586, p<0,001$, respectivamente). Porque são métodos propedêuticos com fun-

\begin{tabular}{|c|rrrrr|}
\hline $\begin{array}{c}\text { Tabela. Concordância entre os campos SWAP e FDT segundo a } \\
\text { gravidade de perda do campo visual glaucomatoso }\end{array}$ \\
$\begin{array}{c}\text { Classificação do campo } \\
\text { blue-yellow }\end{array}$ & Total \\
campo FDT & $\mathbf{4}$ & $\mathbf{1}$ & $\mathbf{2}$ & $\mathbf{3}$ & \\
0 & 2 & 1 & 0 & 0 & 3 \\
1 & 8 & 11 & 3 & 0 & 22 \\
2 & 3 & 4 & 5 & 1 & 13 \\
3 & 1 & 0 & 0 & 4 & 5 \\
Total & 14 & 16 & 8 & 5 & 43 \\
\hline
\end{tabular}

cionamento diferentes, já que o SWAP testa 52 pontos nos 24 graus centrais (24-2), e o FDT utilizado nesse estudo visa apenas 17 pontos nos 20 graus centrais (C-20), é esperado que as estratégias matemáticas para cálculo dos índices globais sejam diferentes. Entretanto, nosso estudo verificou uma associação linear (correlação) direta entre os dois campímetros, ou seja, se os valores do MD ou PSD em uma das estratégias aumenta ou diminui, o mesmo ocorre com a segunda, refletindo comportamento correlato do FDT e do SWAP na determinação do dano glaucomatoso.

Ao se comparar os campos em relação à gravidade da lesão glaucomatosa, evidenciamos uma concordância moderada $(\mathrm{k}=0,319)$, porém altamente significativa $(\mathrm{p}<0,001)$. É possível notar que a maior discrepância ocorre em pacientes com glaucoma inicial (Tabela). Uma possível explicação para o fato relaciona-se ao efeito aprendizado do FDT. Apesar de todos os pacientes incluídos terem sido submetidos a um exame de "screening" prévio, os pacientes eram inexperientes com o FDT, e com isso a ocorrência de resultados falso-positivos pode ser esperada $^{(13)}$. Entretanto, todos os pacientes já haviam realizados pelo menos dois exames de campo visual. Com isso, acreditamos que os resultados da perimetria azul-amarelo sejam mais confiáveis.

Algumas explicações podem ser encaixadas no caso. Como já foi sugerido em estudo anterior, é possível que diferentes subpopulações celulares sejam lesadas em pessoas diferentes no glaucoma ${ }^{(17)}$. Dessa forma, ao se analisar uma amostra relativamente importante (43 olhos), estaríamos examinando pacientes cujo dano glaucomatoso tenha inicialmente abordado as células magnocelulares, e outros onde a camada parvocelular foi lesada primeiro. Com isso, na análise final os pacientes poderiam comportar-se de maneira semelhante. Entretanto, os níveis da correlação não estariam altos como no presente estudo, o que praticamente inviabiliza essa hipótese. Além disso, podemos também postular que não haja seletividade no dano glaucomatoso precoce para as células magnocelulares ou parvocelulares, ou que os instrumentos disponíveis não sejam capazes de isolar as células ganglionares de forma completa.

Como limitações do estudo, temos a inclusão de ambos os olhos de alguns pacientes, que pode influenciar os resultados finais pelo fato de que pode existir uma correlação inter-ocular entre as medidas dos dois olhos de cada indivíduo. Além disso, uma amostra mais apropriada para avaliar o dano seletivo incluiria apenas pacientes com dano inicial (apesar do dano médio ser limítrofe entre o inicial e o moderado $\left.{ }^{(15)}\right)$, onde teoricamente esse dano ocorre, e em uma análise setorial, para evitar que alterações semelhantes, mas em diferentes regiões do campo visual sejam comparadas e classificadas como equivalentes.

\section{CONCLUSÃO}

De um modo geral, podemos concluir que existe alta correlação entre os índices globais da perimetria azul-amarelo e da perimetria de freqüência dupla, sugerindo a não-seletividade para a perda glaucomatosa inicial, ou a ineficiência dos meios 
diagnósticos atuais de avaliar isoladamente as células ganglionares retinianas. Trabalhos corrigindo as limitações do presente estudo são necessários para confirmar os resultados aqui encontrados.

\section{ABSTRACT}

Purpose: To test the agreement and correlation between the frequency doubling technology and the short wavelength perimetry in glaucoma. Methods: Glaucoma patients were selected from the Glaucoma Sector of CEROF - Universidade Federal de Goiás, and then underwent frequency doubling technology and short wavelength perimetry examinations in both eyes (if eligible) on the same day in a random sequence. Pearson's correlation between the global indices (mean deviation - MD e pattern standard deviation - PSD) and the agreement between the examinations (Kappa) were obtained. Results: Forty-three eyes from 26 patients were included in the study. Comparing the MD $(-4.5 \pm 4.5 \mathrm{~dB}$ for the frequency doubling technology and $-8.0 \pm 6.8 \mathrm{~dB}$ for the short wavelength perimetry, $\mathrm{p}<0.001)$ and PSD $(6.4 \pm 2.8 \mathrm{~dB}$ for the FDT and $5.8 \pm 2.4 \mathrm{~dB}$ for SWAP, $\mathrm{p}=0.1$ ), only MD was statistically different between the groups. The global indices MD e PSD were highly correlated between the frequency doubling technology and short wavelength perimetry $(r=0.644, p<0.001$ and $\mathrm{r}=0.586, \mathrm{p}<0.001$, respectively). There was a high agreement between the examinations (Kappa=0.319, p<0.001). Conclusion: In the present study, a high correlation between the global indices (MD and PSD) by the frequency doubling technology and short wavelength perimetry was found, as well as a high agreement between the examinations. These observations indicate either that both cell populations are similarly affected by glaucomatous damage or that both methods measure activity in the same cell populations.

Keywords: Glaucoma/diagnosis; Perimetry/methods; Ocular hypertension; Comparative studies

\section{REFERÊNCIAS}

1. Kerrigan-Baumrind LA, Quigley HA, Pease ME, Kerrigan DF, Mitchell RS. Number of ganglion cells in glaucoma eyes compared with threshold visual field tests in the same persons. Invest Ophthalmol Vis Sci. 2000;41(3):741-8.

2. Quigley HA, Addicks EM, Green WR. Optic nerve damage in human glaucoma. III. Quantitative correlation of nerve fiber loss and visual field defect in glaucoma, ischemic neuropathy, papilledema, and toxic neuropathy. Arch Ophthalmol. 1982;100(1):135-46.

3. Johnson CA, Adams AJ, Casson EJ, Brandt JD. Progression of early glaucomatous visual field loss as detected by blue-on-yellow and standard white-on-white automated perimetry. Arch Ophthalmol. 1993;111(5):651-6.

4. Johnson CA, Adams AJ, Casson EJ, Brandt JD. Blue-on-yellow perimetry can predict the development of glaucomatous visual field loss. Arch Ophthalmol. 1993;111(5):645-50.

5. Maddess T, Goldberg I, Dobinson J, Wine S, James AC. Clinical trials of the frequency doubled illusions an indicator of glaucoma [abstract]. Invest Ophthalmol Vis Sci. 1995(36):S335.

6. Brusini P, Busatto P. Frequency doubling perimetry in glaucoma early diagnosis. Acta Ophthalmol Scand Suppl. 1998(227):23-4.

7. Burnstein Y, Ellish NJ, Magbalon M, Higginbotham EJ. Comparison of frequency doubling perimetry with Humphrey visual field analysis in a glaucoma practice. Am J Ophthalmol. 2000;129(3):328-33.

8. Cello KE, Nelson-Quigg JM, Johnson CA. Frequency doubling technology perimetry for detection of glaucomatous visual field loss. Am J Ophthalmol. 2000;129(3):314-22.

9. Quigley HA. Identification of glaucoma-related visual field abnormality with the screening protocol of frequency doubling technology. Am J Ophthalmol. 1998;125(6):819-29.

10. Sponsel WE, Arango S, Trigo Y, Mensah J. Clinical classification of glaucomatous visual field loss by frequency doubling perimetry. Am J Ophthalmol. 1998;125(6):830-6.

11. Martin L, Wanger P, Vancea L, Gothlin B. Concordance of high-pass resolution perimetry and frequency-doubling technology perimetry results in glaucoma: no support for selective ganglion cell damage. J Glaucoma. 2003;12(1):40-4.

12. Hudson C, Wild JM, O'Neill EC. Fatigue effects during a single session of automated static threshold perimetry. Invest Ophthalmol Vis Sci. 1994;35(1): 268-80.

13. Schimiti RB, Costa VP, Freitas TG, Magacho L, Arcieri ES, Kara-José N. Curva de aprendizado e flutuações a curto e longo prazos com perimetria de frequência dupla (FDT). Arq Bras Oftalmol. 2003;66(3):299-304

14. Anderson DR, Patella VM. Automated Static Perimetry. 2 ${ }^{\text {nd }}$. St. Louis, Missouri, USA; 1999.

15. Hodapp E, Parrish II RK, Anderson DR. Clinical decisions in glaucoma. St Louis, Missouri: Year Book; 1993.

16. Varma R, Spaeth GL. The optic nerve in glaucoma. Philadelphia: J. B. Lippincott; 1993.

17. Sample PA, Bosworth CF, Blumenthal EZ, Girkin C, Weinreb RN. Visual function-specific perimetry for indirect comparison of different ganglion cell populations in glaucoma. Invest Ophthalmol Vis Sci. 2000;41(7):1783-90.

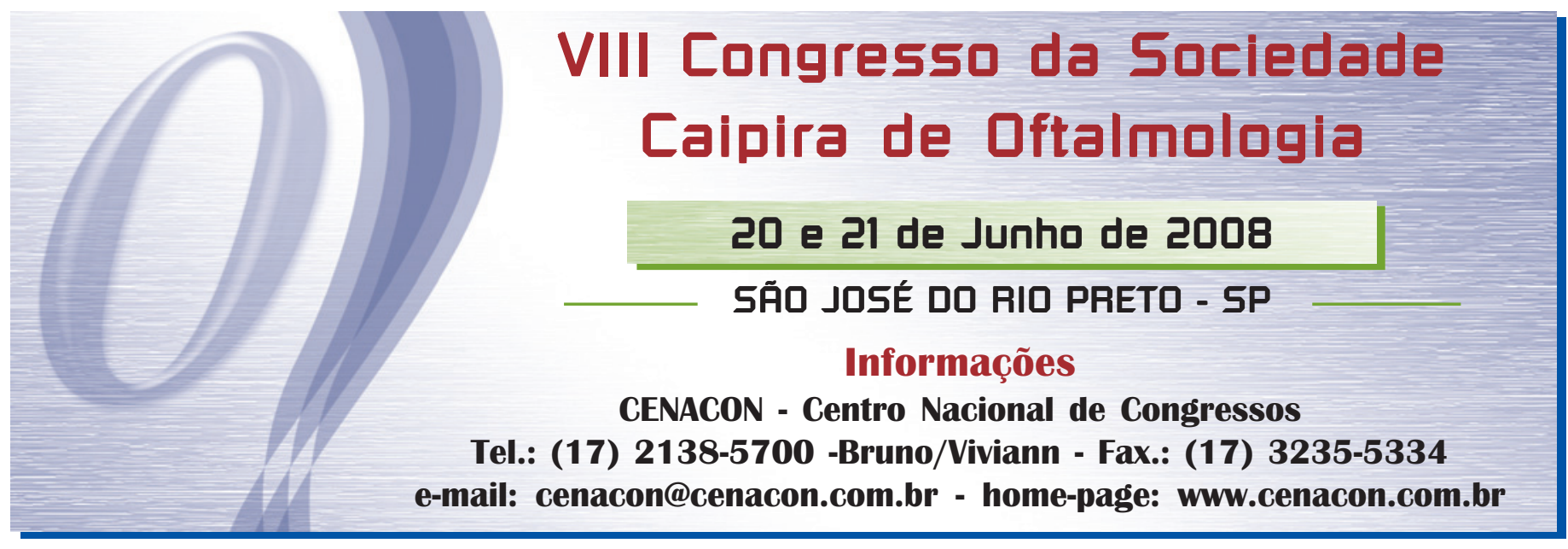

